Meta

Journal des traducteurs

Translators' Journal

\title{
Les conquérants espagnols et les terminologies indo-américaines
}

\section{Véronique Huyngh-Armanet}

Volume 34, numéro 3, septembre 1989

1. Actes du Colloque Les terminologies spécialisées : Approches quantitative et logico-sémantique et 2 . Actes du Colloque Terminologie et Industries de la langue

URI : https://id.erudit.org/iderudit/001901ar

DOI : https://doi.org/10.7202/001901ar

Aller au sommaire du numéro

Éditeur(s)

Les Presses de l'Université de Montréal

ISSN

0026-0452 (imprimé)

1492-1421 (numérique)

Découvrir la revue

Citer cet article

Huyngh-Armanet, V. (1989). Les conquérants espagnols et les terminologies indo-américaines. Meta, 34(3), 558-566. https://doi.org/10.7202/001901ar
Résumé de l'article

Les chroniques des Indes ont intégré, inégalement il est vrai, un certain nombre de termes empruntés aux langues amérindiennes du XVIe siècle. Traditionnellement, ces terminologies sont le plus souvent étudiées d'un point de vue historico-étymologique. À partir d'une de ces descriptions, comportant des recensements textuels exhaustifs, nous esquissons un ensemble de propositions destinées à faciliter l'analyse de ces terminologies, en dépit du caractère composite des textes qui leur servent de vecteurs. 


\title{
LES CONQUÉRANTS ESPAGNOLS ET LES TERRMINOLOGIES INDO-AMÉRICAINES
}

\author{
VÉRONIQUE HUYNH-ARMANET \\ Responsable du laboratoire d'analyse relationnelle des textes \\ Université de Paris VIII, Paris, France
}

RÉSUMÉ

Les chroniques des Indes ont intégré, inégalement il est vrai, un certain nombre de termes empruntés aux langues amérindiennes du XVI ${ }^{\mathrm{e}}$ siècle. Traditionnellement, ces terminologies sont le plus souvent étudiées d'un point de vue historico-étymologique. À partir d'une de ces descriptions, comportant des recensements textuels exhaustifs, nous esquissons un ensemble de propositions destinées à faciliter l'analyse de ces terminologies, en dépit du caractère composite des textes qui leur servent de vecteurs.

\section{LES TERMINOLOGIES INDO-AMÉRICAINES DANS LE CONTEXTE TECHNOLOGIQUE ACTUEL}

Le développement rapide des technologies actuelles est en train de modifier, non seulement les terminologies qui les décrivent, mais, dans une large mesure, l'approche même, des ensembles terminologiques à décrire y compris la description des terminologies exotiques du passé.

Pour celles dont nous pouvons suivre d'assez près la genèse - c'est le cas en particulier des terminologies indo-américaines qui se sont constituées essentiellement dans les trois décades ayant suivi la découverte du Nouveau Monde - il semble de plus en plus difficile de se contenter d'explorations historico-étymologiques, même si de telles explorations constituent dans la majorité des cas, un passage obligé. Il paraît indispensable, en effet, chaque fois que l'occasion en est fournie, d'étudier les termes dans leur double contextualité essentielle: syntaxique bien sûr, mais aussi lorsqu'il s'agit de documents à structure complexe, structurelle; ceci au nom de la plasticité intrinsèque des langues dont le respet a orienté, dès nos premiers travaux, nos méthodologies descriptives ${ }^{1}$. Nous sommes malheureusement très loin encore, de pouvoir présenter une description génétique contextuelle de ces terminologies exotiques, même si une telle description semble théoriquement réalisable, essentiellement à travers les chroniques des Indes publiées en espagnol, italien, portugais et même en latin dans la première moitié du XVIe siècle. Il y faudrait cependant des collaborations convergentes, à certains égards comparables à celles qui ont donné naissance à ces ensembles terminologiques. C'est pourquoi nous nous bornerons ici plus modestement, à en esquisser l'approche, à partir d'une des chroniques espagnoles marquantes de l'époque, l'Histoire Générale et Naturelle des Indes de Gonzalo Fernandez de Oviedo y Valdés dont le premier Tome parut à Séville en 1535.

\section{REMARQUES PRÉLIMINAIRES}

Ce texte volumineux dont nous ne possédons pas encore, à ma connaissance, de copie magnétique, peut être au centre de notre réflexion parce qu'il a donné lieu non seu- 
lement à un inventaire exhaustif hiérarchique des termes amérindiens qu'il contient ${ }^{2}$, mais parce que nous avons pu obtenir, au cours de la première phase, essentiellement historico-étymologique de cette recherche, un recensement des définitions proposées par Gonzalo Fernandez de Oviedo dans cet ouvrage et que grâce à cet administrateur-écrivain, nous commençons à entrevoir concrètement ce qu'a pu être la mutation qui a suivi la découverte du Nouveau Monde 3 .

\subsection{GONZALO FERNANDEZ DE OVIEDO (1478-1557) : UN TÉMOIN PRIVILÉGIÉ}

La chronique sur laquelle nous appuierons nos propositions, est l'une des rares chroniques espagnoles des Indes publiées, fut-ce partiellement avant 1550 . Nous la devons à un écrivain que nous définirions aujourd'hui, comme un homme de terrain proche du pouvoir. Gonzalo Fernandez de Oviedo y Valdès fut en effet, historiographe du Roi. Il entra dès sa prime jeunesse en contact avec la Cour d'Espagne où il servit comme page; il avait 14 ans au moment de la découverte des Indes par Christophe Colomb.

Il ne fut pas comme Hernan Cortés (1495-1547) un Grand Capitaine; mais certainement un grand voyageur. Selon Roger Valensi, il se rendit aux Antilles pour la première fois en 1514 (il avait déjà 36 ans), comme «Contrôleur de la fonte de l'or»; il y retourna ensuite une douzaine de fois. Il y séjourna à maintes reprises longuement et, selon certaines souces, y mourut. Il était encore à près de 80 ans Gouverneur de Saint-Domingue.

L'histoire générale et naturelle des Indes semble avoir occupé une grande place dans sa vie. Il l'annonça en quelque sorte par un texte beaucoup plus court: le Sumario de la Natural Historia en Las Indias, publié à Tolède en 1526 et se rendit en Espagne en 1535 pour la parution du premier des cinq tomes de l'Histoire Générale; le seul à avoir été publié de son vivant...

Considéré comme un historiographe autodidacte, il eut de nombreux détracteurs. À notre époque, on lui reproche encore souvent d'être confus; ce qui signifie pour nous qu'il ne faut pas l'aborder sans précautions. C'est pour cela que nous avons programmé une exploration morpho-syntaxique assistée par ordinateur d'un corpus expérimental d'énoncés ${ }^{4}$ appartenant au sous-ensemble terminologique dit des Éléments Naturels ${ }^{5}$. C'est également autour de ce sous-ensemble que nous articulerons une partie importante de nos propositions méthodologiques. Toutefois, il nous paraît souhaitable d'examiner quelques-unes des informations quantitatives dont nous disposons déjà sur cette chronique et grâce à elles, de mettre en parallèle les termes communs à l'ensemble terminologique d'Oviedo et ceux des termes de cet ensemble qui continuent de vivre dans notre français actuel.

\section{QUELQUES INFORMATIONS QUANTITATIVES INDISPENSABLES}

Nous les rechercherons successivement à travers les relevés hiérarchiques exhaustifs, les relevés par langues-sources et les relevés thématiques provisoires qui nous sont proposés par Roger Valensi.

\subsection{LES RELEVÉS HIÉRARCHIQUES}

Parce que ces relevés sont, comme nous l'avons indiqué précédemment, avant tout orientés vers l'identification des langues auxquelles ont été empruntés les termes indoaméricains retenus par Fernandez de Oiedo, le repérage sous des graphies multiples d'un même terme a été conduit avec soin. Le nombre de termes proposé par Valensi (342), quels que soient les risques des relevés manuels est certainement assez juste, ainsi d'ailleurs que le nombre d'hapax : 118 .

Les termes de fréquence supérieure à un sont d'après ces inventaires, très inégalement répartis dans ce texte puisque nous avons, par exemple, 1117 occurrences pour 
cacique, 518 pour canoa, 378 pour maiz, que nous passons en dessous de la barre des 100 occurrences avec le sixième terme, yucca, en dessous de la barre des 50 avec le treizième terme, de celle des vingt avec le $35^{\mathrm{e}}$ terme : batata, etc.

3.1.1 La sélection dans ces index hiérarchiques des termes indo-américains utilisés par le français courant actuel montre la disparité quantitative des deux ensembles. Nous avons sélectionné vingt termes dont dix-neuf figurent dans le dictionnaire Robert de 1981, et un barbecue, dans son supplément. Notons cependant que le terme cacique, de loin le plus fréquent chez Oviedo, s'emploie surtout de nos jours en français actuel d'une part sous la forme d'un tableau et d'autre part sous la forme d'un histogramme représentatif de la fréquence des termes communs dans l'œuvre espagnole examinée.

3.1.2 Dans le tableau, figurent en lignes, de gauche à droite: le rang du terme, toujours selon Roger Valensi, le terme lui-même, son nombre d'occurrences dans l'Histoire Générale et Naturelle des Indes, l'équivalent français autre qu'a pour nous ce terme actuellement. Ainsi, dans notre vie quotidienne, le terme yucca désigne non plus comme dans Oviedo le manioc mais une plante d'appartement actuellement à la mode et nous parlons moins de goyavier que de goyave, depuis que ce fruit, le plus souvent sous la forme confite, est entré dans notre alimentation.

3.1.3 L'histogramme n'a d'autre objet que de rendre tangible, la distribution quantitative dans le texte d'Oviedo des termes amérindiens qui se retrouvent dans notre français actuel.

TABLEAU 1

Termes communs à la Historia General y Natural de las Indias et au Français courant actuel

\begin{tabular}{|l|l|c|l|l|}
\hline Rang & \multicolumn{1}{|c|}{ Terme } & Nbre Occ. & \multicolumn{1}{|c|}{ Traduction 1 } & Traduction 2 \\
\hline 1 & CACIQUE & 1117 & Roi, Roitelet. & \\
\hline 2 & CANOA & 518 & Canoë. & \\
\hline 3 & MAIZ & 379 & Maïs. & \\
\hline 5 & INGA & 151 & Inca. & \\
\hline 6 & YUGA & 88 & (Manioc) & Yucca. \\
\hline 8 & SABANA & 72 & Savane. & \\
\hline 9 & CACAO & 67 & (Cacaoyer) & Cacao. \\
\hline 10 & HAMACA & 61 & Hamac. & \\
\hline 14 & HURACAN & 40 & Ouragan. & \\
\hline 17 & BARBACOA & 32 & (Claie en bois) & Barbecue. \\
\hline 25 & PIRAGUA & 29 & Pirogue. & \\
\hline 24 & GUAYABO & 30 & (Goyavier) & Goyave. \\
\hline 34 & IGUANA & 20 & Iguane. & \\
\hline 35 & BATATA & 19 & Patate douce. & \\
\hline 46 & TABAC & 17 & Tabac. & \\
\hline 81 & ANON & 7 & Anone. & \\
\hline 179 & COCA & 2 & Coca. & \\
\hline 206 & PATATA & 2 & (Pomme de terre) & Patate. \\
\hline 229 & AGUACATE & 1 & (Avocatier) & Avocat. \\
\hline 242 & CAIMAN & 1 & Caiman. & \\
\hline
\end{tabular}

N.B. Lorsque le français actuel propose une traduction différente de celle de Gonzalo Fernandez de Oviedo y Valdes, elle figure dans la colonne Traduction 2. 


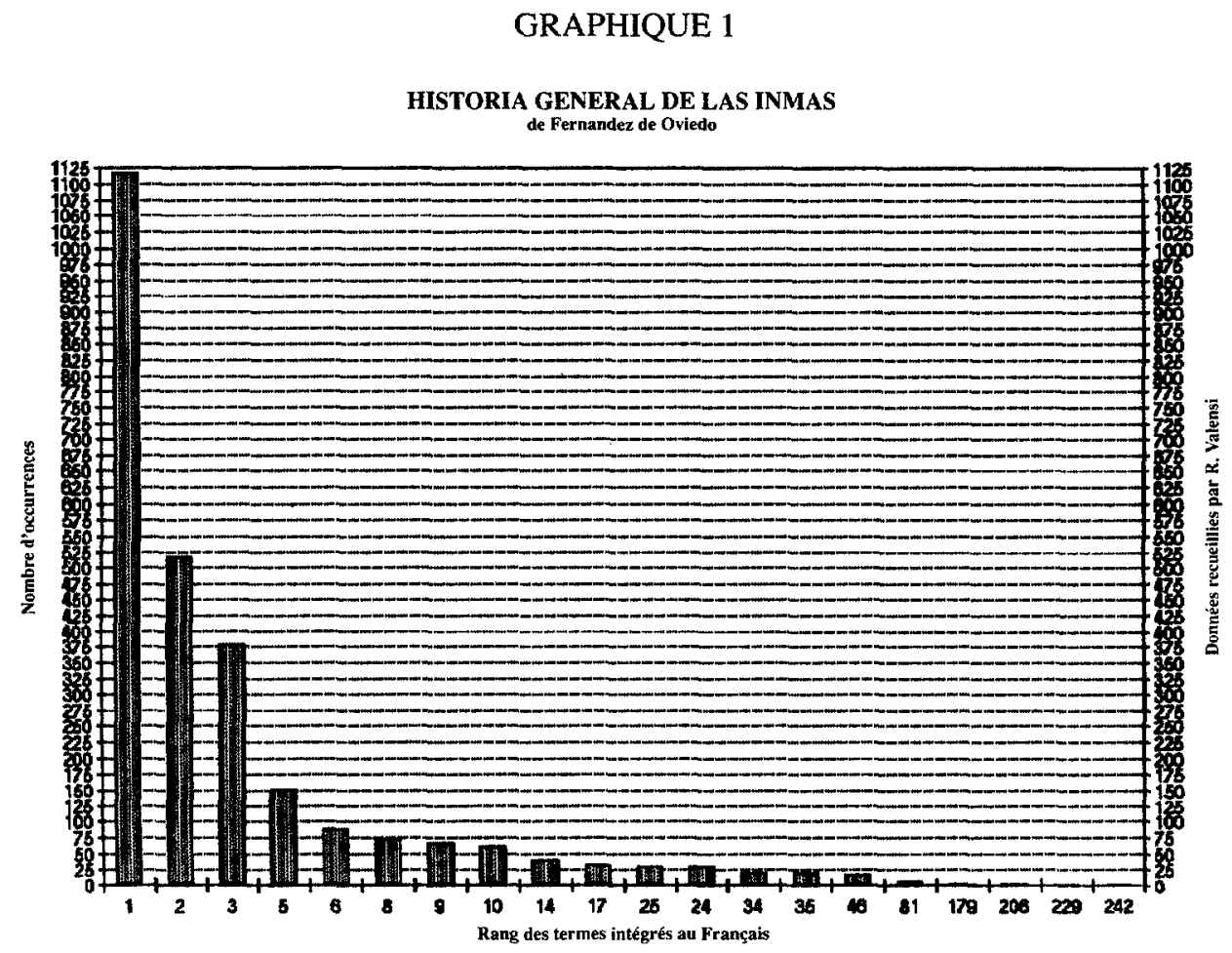

\subsection{LES RELEVÉS PAR LANGUES-SOURCES}

Bien qu'ils soient encore incomplets, ils ont mis en évidence la part prépondérante globale de l'Arawak, dans la terminologie d'Oviedo, et l'existence d'équilibres différents si on constitue des glossaires thématiques de base tels que FAUNE, FLORE, SOCIÉTÉ INDIGENE, ÉLEMENTS NATURELS ...

Nous reprendrons ultérieurement, à propos de l'examen du sous-ensemble dit des ÉLÉMENTS NATURELS les informations fiables que peuvent fournir ces relevés. Notons dès maintenant que pour la sélection amérindienne française, la langue-source dominante est également l'Arawak qui est pour nous une langue-fossile.

\subsection{LES RELEVÉS THÉMATIQUES}

Il s'agissait à l'origine, de relevés tabulaires manuels destinés à concilier les impératifs primordiaux d'une recherche historico-étymologique sur inventaires textuels exhaustifs et ceux de la préparation à travers les lectures attentives imposées par ces inventaires, d'une étude contextuelle ultérieure des termes indo-américains retenus par Oviedo. Pour essayer d'apprécier la distribution thématique des termes, abstraction faite ici des nombres d'occurrences, nous avons représenté graphiquement les ensembles thématiques de base proposés par Roger Valensi et parallèlement ceux qui peuvent être réalisés à partir de nos vingt termes, selon les mêmes critères; c'est-à-dire sans prendre en 
compte la contextualité. Ces graphiques sont à interpréter comme des documents à confronter ultérieurement avec ceux que nous risquons d'obtenir, une fois intégrées l'ambiguïté de certains termes de la terminologie indo-américaine de Gonzalo Fernandez de Oviedo. C'est à une expérience de désambiguïsation que nous allons maintenant nous attacher à partir d'un sous-ensemble de très faible effectif (treize termes), celui qui figure dans l'étude de Roger Valensi sous le nom «ÉLÉMENTS NATURELS».

\section{GRAPHIQUE 2}

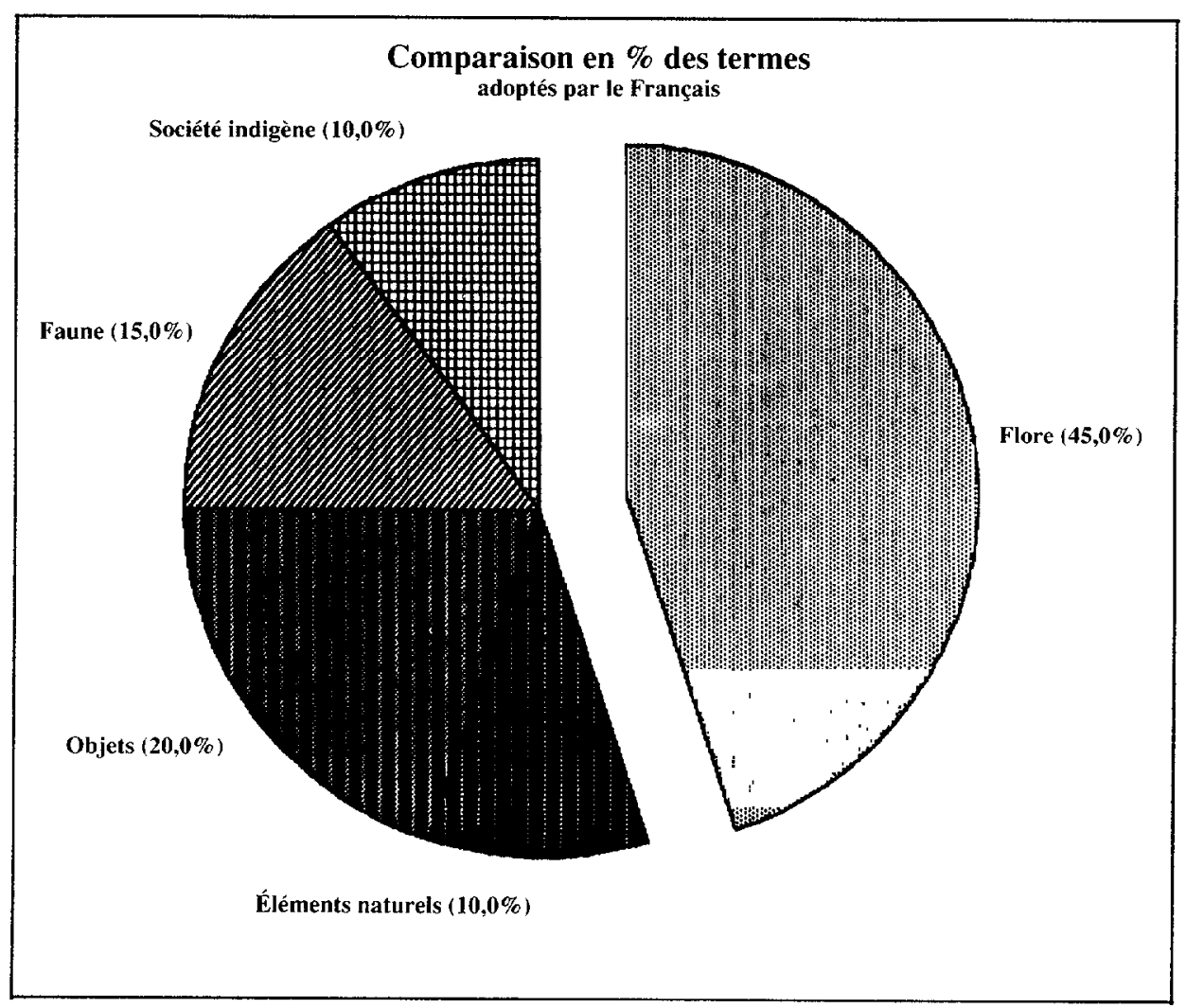


GRAPHIQUE 3

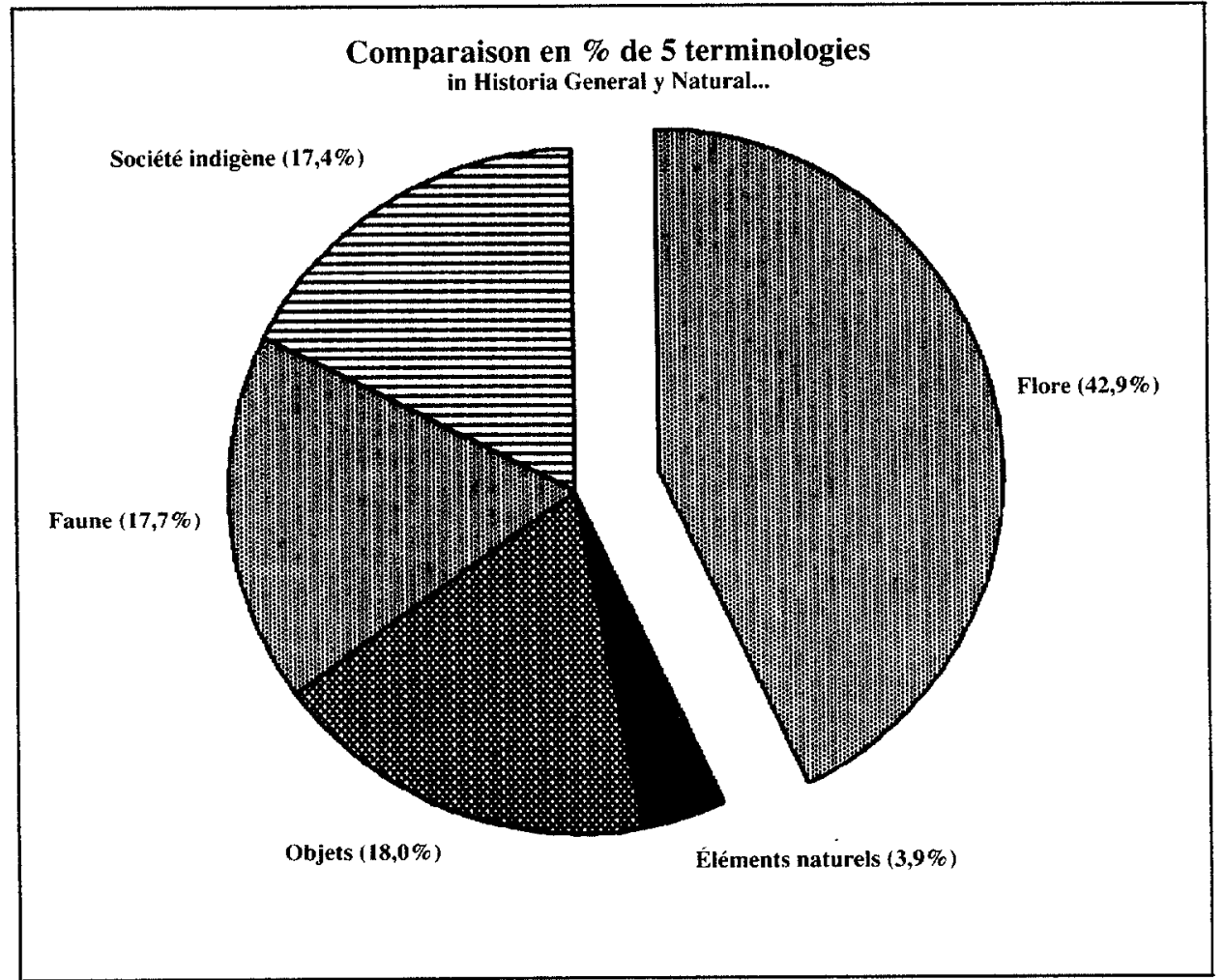

\section{AMORCE D'UNE ÉTUDE CONTEXTUELLE DU SOUS-ENSEMBLE DIR} DES ÉLÉMENTS NATURELS

Pour engager cette étude, nous avons, à partir de la table initiale des Éléments Naturels, et des définitons proposées par Oviedo pour chacun de ces termes, constitué informatiquement, un nouveau modèle de table où figurent successivement, en ligne: la forme atestée chez Oviedo, sa traduction, le nombre d'occurrences, les langues-sources quand elles ont pu être identifiées, les associations signalées par Oviedo, la traduction des formes associées, leur champ d'application initial éventuel Terme 1) et leur champ d'application post-colombien. 
TABLEAU 2

Terminologie dite des Éléments Naturels

\begin{tabular}{|c|c|c|c|c|c|c|c|}
\hline Forme & $\begin{array}{l}\text { Traduc- } \\
\text { tion }\end{array}$ & $\begin{array}{l}\text { Nbre } \\
\text { Occ. }\end{array}$ & $\begin{array}{c}\text { L. } \\
\text { sources }\end{array}$ & $\begin{array}{c}\text { Asso- } \\
\text { ciation }\end{array}$ & $\begin{array}{l}\text { Traduc- } \\
\text { tion }\end{array}$ & Terme 1 & Terme 2 \\
\hline bagua & mer & 2 & arawak & & & & \\
\hline $\begin{array}{l}\text { chia } \\
\text { (echia) }\end{array}$ & lune & 1 & autres.. & & & & \\
\hline chisse & soleil & 1 & quechua & & & & \\
\hline cocha & mer & 1 & quechua & & & & \\
\hline guatan & tourbillon & 1 & quechua & & & Divinité & \\
\hline huracan & ouragan & 40 & arawak & & & & \\
\hline jaguey & mare & 22 & arawak & & & & \\
\hline parana & mer & 1 & tupi-guarani & /guazï & & & \\
\hline pechry & mer & 1 & autres.. & & & & \\
\hline quin & soleil & 1 & maya & al/ & fils du soleil & $\begin{array}{l}\text { Nom de } \\
\text { prêtre }\end{array}$ & $\begin{array}{l}\text { nom des } \\
\text { espagnols }\end{array}$ \\
\hline sabana & sabane & 72 & arawak & & & & \\
\hline usa & soleil & 2 & autres.. & /chia & $\begin{array}{l}\text { soleil } \\
\text { et lune }\end{array}$ & Divinité & $\begin{array}{l}\text { nom des } \\
\text { espagnols }\end{array}$ \\
\hline vira & écume & 1 & quechua & /cocha & $\begin{array}{l}\text { écume } \\
\text { de la mer }\end{array}$ & & $\begin{array}{l}\text { nom des } \\
\text { espagnols }\end{array}$ \\
\hline
\end{tabular}

\subsection{LES TERMES APPAREMMENT SUPERFLUS}

Cette liste comporte un certain nombre de termes apparemment superflus, si nous considérons comme on le fait généralement que l'introduction des terminologies amérindiennes correspond à la nécessité de décrire un nouvel univers.

Ainsi, à côté de termes correspondant à de nouvelles réalités: sabana, la savane; huracan, l'ouragan; jaguey, le point d'eau et peut-être guatan, le tourbillon, nous trouvons quatre termes dont un seul est Arawak, pour désigner la mer; trois désignant le soleil et même un terme pour désigner l'écume de la mer. On note parallèlement que les termes autres que sabana, huracan et jaguey sont généralement ou des hapax ou des termes à très basse fréquence. D'autre part, certains d'entre eux sont présentés par Oviedo comme associés: al-quin; parana-guazu; usachies; vira-cocha. Il est évident qu'à travers trois de ces quatre associations surgissent conjointement les mythes précolombiens et la vision qu'ont eue des espagnols les indiens décrits par Oviedo. La terminologie semble donc nous entraîner au-delà de ses limites initiales.

\subsection{LES TERMES «UTILES»}

Reste le problème des termes à haute fréquence essentiellement destinés à définir un univers autre, à savoir savane, ouragan et jaguay. Si nous nous limitions aux définitions, nous devrions noter qu'elles sont dans ces trois cas, assez précises et parfois tout à fait proches de celles de notre lexicographie actuelle. Ainsi, Oviedo définit sabana en des 
termes tout à fait comparables à ceux qu'utilise de nos jours Le Robert pour savane. Avec jaguey, nous avons même droit à une description presque technique sur la création par les autochtones de points d'eau soit près de la mer, soit près d'une rivière. Le terme est également donné comme un équivalent de «mare». Ce terme intéressant à confronter avec les autres survivances en espagnol des terminologies amérindiennes du XVIe siècle n'a pas été intégré au français. Nous ne nous y attarderons pas ici.

On pourrait noter en revanche, qu'Oviedo omet de signaler, dans sa définition de l'ouragan - comparable, selon lui, à une tempête de très grande violence - l'existence de vents contraires. Sauf si on admet que dans un ouvrage qui n'est pas un dictionnaire, la définition peut avoir une autre typologie que celle que requiert la lexicographie et qu'il faut s'attendre à des définitions, soit éclatées, soit illustrées. Et de fait, nous retrouvons l'information relative aux vents contraires à travers la description d'un des ouragans dont Oviedo fut le témoin en suivant les vaisseaux qui sont arrachés à leurs amarres pour revenir se briser sur la côte.

4.3 Que conclure de ce premier parcours inachevé puisque nous n'avons pas encore fini d'étudier en contexte les occurrences des trois termes les plus fréquemment utilisés?

4.3.1 Qu'on aurait certainement le plus grand intérêt à appliquer à des textes comme cette chronique de Gonzalo Fernandez de Oviedo, les techniques d'enregistrement intégral qui permettraient la réalisation de concordances terminologiques exhaustives.

4.3.2 Mais qu'il faudrait, pour ne pas commettre trop d'erreurs dans l'étude de ces textes où se mêlent par exemple, le récit d'actualité, l'évocation d'entretiens, les rappels historiques et les considérations économiques, utiliser des logiciels dits plurilinguistiques, permettant par une pré-édition structurelle adéquate 6 de poser les jalons indispensables à la définition de blocs structurellement homogènes.

\section{REMARQUES TERMINALES}

Plus nous progressons dans l'étude de textes aussi importants à la fois pour la connaissance de nos langues et de mutations vraisemblablement aussi brutales que celles que nous connaissons, mieux nous mesurons ce qu'il pourrait y avoir de «barbare» à entrer dans ces textes sans utiliser les meilleures des technologies à notre disposition pour tenter de comprendre, en particulier à travers l'insertion de telles terminologies, une partie du non-dit du discours de la découverte du Nouveau Monde. Comme un certain nombre de textes techniques modernes mais sans doute plus qu'eux, de telles œuvres appellent donc l'alliance intime des recherches terminologiques et cognitives.

Dans un univers où les problèmes de rentabilité paraissent souvent primordiaux, il est certes légitime de s'interroger sur la rentabilité immédiate de telles recherches. Sauf peut-être si on se souvient que dans les échanges inter-linguistiques devenus essentiels pour notre époque, nous avons aussi à traiter les textes à structures complexes et que l'apprentissage de ce qui transparaît de l'intégration des mots, est plus que jamais culturellement essentiel.

NOTES

1. Références bibliographiques

HUYNH-ARMANET, V. (1976): Recherches sur la structuration syntaxique de l'espagnol contemporain, Lille III et Honoré Champion.

HUYNH-ARMANET, V. (1977): Des structures syntaxiques de l'espagnol à l'analyse relationnelle des textes, Paris, Honoré Champion. 
HUYNH-ARMANET, V., PINEIRA-TRESMONTANT, C. (1986) : «Laboratoire d'analyse relationnelle des textes : présentation des travaux», Computers and the Humanitis, vol. $20, \mathrm{n}^{\circ} 3$, Paradigm Press.

2. Cf. Roger Valensi (1986), les indo-américanismes dans la Historia general y natural de las Indias de Gonzalo Fernandez de Oviedo y Valdés, Thèse de $3^{\mathrm{e}}$ Cycle, Université de Paris VIII.

3. La direction de la recherche historico-étymologique a été assurée par Raymond Marcus dont les travaux dans ce domaine font autorité.

4. Cette recherche essentiellement cognitive a pour objet, au plan linguistique, l'élaboration par voie inductive des modèles qui sous-tendent ce texte et d'en faciliter par-là même l'étude terminologique en situation.

5. Hors-contexte, cette dénomination est tout à fait adéquate.

6. Références bibliographiques

HUYNH-ARMANET, V., PINEIRA-TRESMONTANT, C. (1987) : «Informatique et réflexion linguistique», Actes du Colloque de la Société de linguistique-hispanique, pp. 157-174, Rouen, France.

HUYN-ARMANET, V., PINEIRA-TRESMONTANT, C. (1988): «L'équilibre des rôles dans trois intermèdes de Cervantes, Hommage à M. Molho», Cahiers de Fontenay, pp. 247-260, Paris.

HUYN-ARMANET, V., PINEIRA-TRESMONTANT, C. (1988) : Les fluctuations des déterminants dans trois intermèdes de Cervantes», Hommage à B. Pottier, vol. 1, pp. 361-395.

HUYN-ARMANET, V., PINEIRA-TRESMONTANT, C. (1988) «Linguistique textuelle et traduction : quelques aides à l'interprétation». Congrès de la Société Française des Hispanistes, Caen (1987).

HUYN-ARMANET, V., SANTI, I. (1983) «Propuesta para un estudio informatizado del teatro espanol», El teatro menor en Espana a partir del Siglo XVI, Madrid, CSIC.

PINEIRA-TRESMONTANT, C. (1984) «La constitution espagnole de 1978, Étude linguistique informatisée», Thèse de $3^{e}$ Cycle, Paris III.

SANCHEZ PEREZ, F.J. (1988) : Contribution à l'élaboration d'outils informatiques pour l'interprétation du texte narratif : application à un nouvel exemplaire de Cervantes, Mémoire de Maitrise, Paris VIII. 